\title{
Treatment of intraocular spread of acanthamoeba after tectonic corneal graft in acanthamoeba keratitis
}

\author{
Francisco Arnalich-Montiel ${ }^{1} \cdot$ Maria Reyes-Batlle ${ }^{2} \cdot$ Rogelio López-Vélez $^{3} \cdot$ Jacob Lorenzo-Morales $^{2}$
}

Received: 25 April 2017 / Revised: 29 October 2017 / Accepted: 9 November 2017 / Published online: 23 February 2018

(c) The Royal College of Ophthalmologists 2018

The number of reports on anterior chamber detection of Acanthamoeba by PCR in the course of Acanthamoeba keratitis (AK) has risen, frequently following recent tectonic keratoplasty [1-3]. Topical biguanide or diamidines do not enter the AC [4] and are cytotoxic when used intraocularly. Herein we report the use of intracameral voriconazole, with proven cysticidal activity in AK [5, 6], for intraocular dissemination of Acanthamoeba, in two consecutive patients.

\section{Case reports}

\section{Case 1}

A 56-year-old male with late-diagnosis culture-positive AK in the right eye (3 months from symptom onset) developed, despite the use of combined topical clorhexidine and propamidine, severe melting with corneal perforation requiring a 9-mm PK. Postoperative treatment included topical clorhexidine, moxifloxacin, dexamethasone, oral prednisolone and tacrolimus. An increased Tyndall reaction, mutton fat keratic precipitates and ciliary hyperemia appeared on day 5; vitreous remained clear. An AC tap was positive for T4 Acanthamoeba and $100 \mu \mathrm{g} / 0.1 \mathrm{ml}$ voriconazole was administered intracameral. Oral immunosuppression was stopped and oral voriconazole $200 \mathrm{mg}$ BDS with $1 \%$ topical voriconazole QDS were started. Intracameral voriconazole administration plus AC tap were repeated three days,

Francisco Arnalich-Montiel

farnalich@gmail.com

1 Cornea Unit, Ophthalmology Department, Ramón y Cajal Hospital, Madrid, Spain

2 University Institute of Tropical Diseases of the Canary Islands, University of La Laguna, Tenerife, Spain

3 Tropical Medicine \& Clinical Parasitology, Infectious Diseases Department, Ramón y Cajal Hospital, Madrid, Spain
1 week and 1 month after diagnosis. The last tap confirmed the absence of Acanthamoeba (Fig. 1). At 4 years' follow up, the AC is inflammation free.

\section{Case 2}

A 60-year-old female with late-diagnosis culture-positive $\mathrm{AK}$ in the right eye (4 months from symptom onset) developed, despite the use of combined topical clorhexidine and propamidine, central melting with perforation requiring $9.5 \mathrm{~mm}$ PK. Postoperative treatment was as in previous case. Increased Tyndall reaction and mutton fat keratic precipitates were detected 3 weeks later; vitreous remained clear. An aqueous tap was taken and intracameral voriconazole administered at the same dose as in case 1. Culture and PCR of the AC tap showed T4 Acanthamoeba. Intracameral voriconazole administration and AC tap were repeated 10 days, and 1 month later together with a new limbus-to-limbus graft to treat disease recurrence outside the graft margins. This last tap showed no amoeba in the AC (Fig. 1). Six months after the last graft, it remains clear, with no signs of AC inflammation (Fig. 2).

\section{Comment}

Acanthamoeba spp. rarely progress beyond the cornea due to an intense neutrophil reaction against intraocular trophozoites, although they are capable of passing through Descemet's Membrane in vitro [7]. Tectonic keratoplasty may predispose to intraocular dissemination of the amoeba by means of a temporary disruption of Descemet's Membrane and the use of oral immunosuppression postoperatively, which can jeopardize the innate immune response.

It is probably an underreported complication confounded with sterile inflammation facilitated by corneal tectonic graft, nevertheless, prompt identification in cases with postoperative AC inflammation is paramount. We documented the successful treatment of two cases of 
Case Report 1

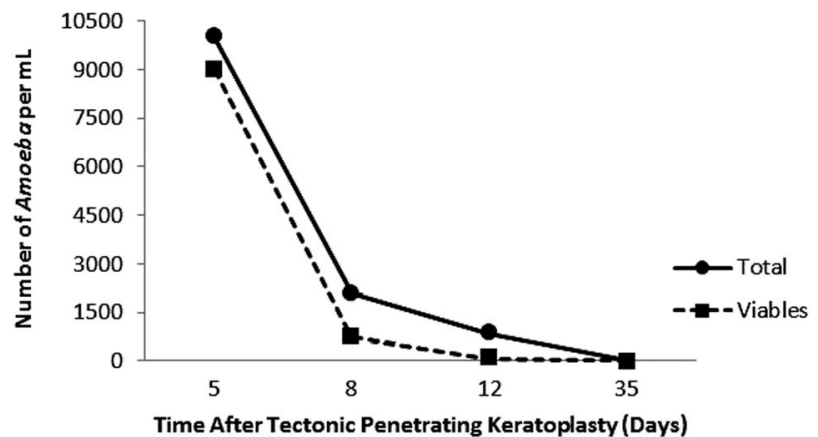

Case Report 2

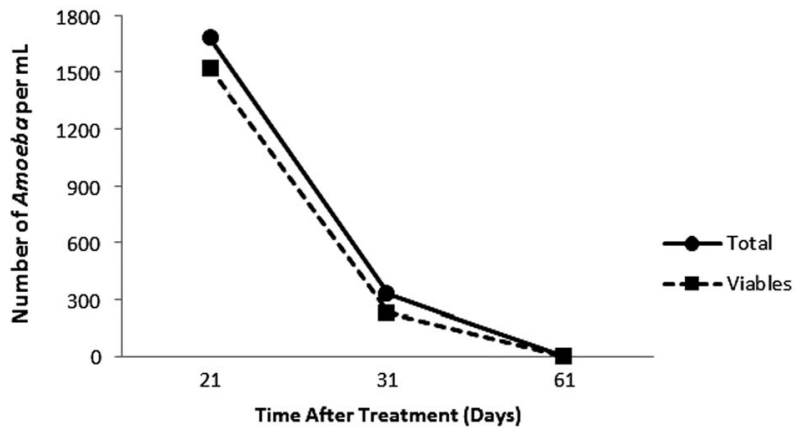

Fig. 1 Evolution of total and viable Acanthamoeba concentrations using direct microscopic examination in an aliquot of $0.1 \mathrm{ml}$ of aqueous humor from the time of the tectonic penetrating keratoplasty to the resolution of the case report 1 and case report 2
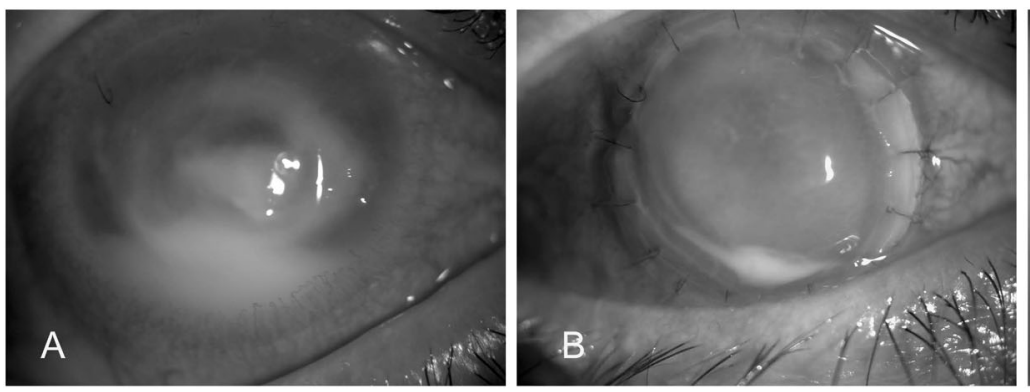

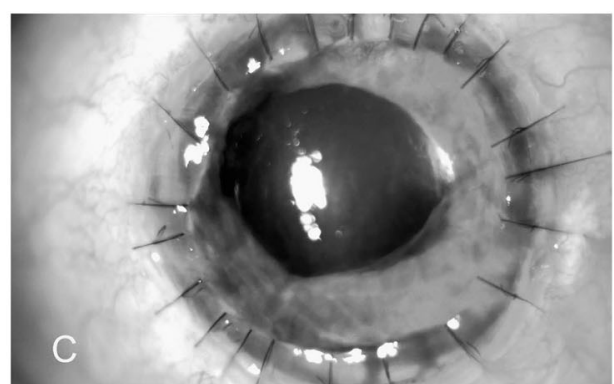

for Acanthamoeba in the aqueous humor (b), and after the second corneal graft with absence of amoeba in the aqueous humor (c)

2. Arnalich-Montiel F, Martin-Navarro CM, Alio JL, Lopez-Velez R, Martinez- Carretero E, Valladares B, et al. Successful monitoring and treatment of intraocular dissemination of acanthamoeba. Arch Ophthalmol. 2012;130:1474-5.

3. Dang Burgener NPL, Baglivo E, Farpour B, Brozou C, Dosso A, Safran A. Acanthamoeba detection in the anterior chamber. Br J Ophthalmol. 2006;90:649-50.

4. Vontobel SF, Abad-Villar EM, Kaufmann C, Zinkernagel AS, Hauser PC, Thiel MA. Cornealpenetrationofpolyhexamethylenebiguanideand chlorhexidine digluconate. J Clin Exp Ophthalmol. 2015;6:430.

5. Bang S, Edell E, Eghrari AO, Gottsch JD. Treatment with voriconazole in 3 eyes with resistant Acanthamoeba keratitis. Am J Ophthalmol. 2010;149:66-69.

6. Martin-Navarro CM, Lopez-Arencibia A, Arnalich-Montiel F, Valladares B, Pinero JE, Lorenzo-Morales J. Evaluation of the in vitro activity of commercially available moxifloxacin and voriconazole eye-drops against clinical strains of Acanthamoeba. Graefes Arch Clin Exp Ophthalmol. 2013;251:2111-7.

7. Davis MJ, Packo KH, Epstein RJ, Grostern RJ, Cohen JA. Acanthamoeba endophthalmitis following penetrating keratoplasty for Acanthamoeba keratitis. Arch Ophthalmol. 2010;128:505-6.

\section{References}

1. Agarwal R, Mittal G, Raj A, Gupta S. Acanthamoeba detection in the anterior chamber after therapeutic penetrating keratoplasty. $\mathrm{J}$ Clin Diagn Res. 2014;8:DD01-2. 